\title{
Observations of foetal heart veins draining directly into the left and right atria
}

\author{
J.H. Kim ${ }^{1}$, O.H. Chai ${ }^{1}$, C.H. Song ${ }^{1}$, Z.W. Jin², G. Murakami ${ }^{3}$, H. Abe ${ }^{4}$ \\ ${ }^{1}$ Department of Anatomy and Institute of Medical Sciences, Chonbuk National University Medical School, Jeonju, Korea \\ ${ }^{2}$ Department of Anatomy, Wuxi Medical School, Jiangnan University, Wuxi, China \\ ${ }^{3}$ Division of Internal Medicine, Jikou-kai Clinic of Home Visits, Sapporo, Japan \\ ${ }^{4}$ Department of Anatomy, Akita University School of Medicine, Akita, Japan
}

[Received: 19 June 2018; Accepted: 8 August 2018]

Evaluation of semiserial sections of 14 normal hearts from human foetuses of gestational age 25-33 weeks showed that all of these hearts contained thin veins draining directly into the atria (maximum, 10 veins per heart). Of the 75 veins in these 14 hearts, 55 emptied into the right atrium and 20 into the left atrium. These veins were not accompanied by nerves, in contrast to tributaries of the great cardiac vein, and were negative for both smooth muscle actin (SMA) and CD34. However, the epithelium and venous wall of the anterior cardiac vein, the thickest of the direct draining veins, were strongly positive for SMA and CD34, respectively. In general, developing fibres in the vascular wall were positive for CD34, while the endothelium of the arteries and veins was strongly positive for the present DAKO antibody of SMA. The small cardiac vein, a thin but permanent tributary of the terminal portion of the great cardiac vein, was also positive for SMA and CD34. A few S100 protein-positive nerves were observed along both the anterior and small cardiac veins, but no nerves accompanied the direct drainage veins. These findings suggested that the latter did not develop from the early epicardiac vascular plexus but from a gulfing of the intratrabecular space or sinus of the atria. However, the immunoreactivity of the anterior cardiac vein suggests that it originated from the vascular plexus, similar to tributaries of the great cardiac vein. (Folia Morphol 2019; 78, 2: 283-289)

Key words: great cardiac vein, anterior cardiac vein, venae cordis minimae, smooth muscle actin, CD34, human foetuses

\section{INTRODUCTION}

Most cardiac veins drain into the right atrium via the coronary sinus. Of these veins, the great cardiac vein is always thickest and has the largest area of drainage [13]. The small cardiac vein is a tributary of the great cardiac vein or the coronary sinus. These veins develop from the epicardiac vascular plexus $[15,16]$. In contrast, the other thin veins, including the venae cordis minimae or the smallest cardiac veins, drain directly into the atrial and ventricular cavities, although the definition of the venae cordis minimae has varied among researchers (reviewed by von Lüdinghausen et al. [17]). Of the latter group of veins, the anterior cardiac vein is always thickest and drains directly into the right atrium. The terminal to the atrium exhibits individual variations, independent of tributaries of the great cardiac vein, or one of the branches joins the peripheral portion of the great

Address for correspondence: J.H. Kim, BS, PhD, Department of Anatomy, Chonbuk National University Medical School, 20 Geunji-ro, Deokjin-gu, Jeonju, 54907, Republic of Korea, tel: 82-63-270-3097, fax: 82-63-274-9880, e-mail: 407kk@hanmail.net 
cardiac vein $[9,18]$. These veins, which are independent of the coronary sinus, are believed to develop from the embryonic intertrabecular spaces along the internal aspects of the developing cardiac walls $[3,4]$. The intertrabecular spaces correspond to the intramural venous sinuses or tunnels in adults $[12,18]$. Alternatively, Mechanik [8] stated that all cardiac veins may develop from the epicardiac vascular plexus, although no linedrawings or photographic evidence was presented. The present study was therefore designed to determine whether foetal thin veins drain directly into the left and right atria. For comparison, the anterior cardiac vein and small cardiac vein were visualised in late-stage foetuses.

\section{MATERIALS AND METHODS}

The study was performed in accordance with the provisions of the Declaration of Helsinki 1995 (as revised in 2013). Sagittal sections were obtained from 14 normal hearts of human foetuses of gestational age 25-33 weeks (crown-rump length [CRL], 200-280 mm). These foetuses were parts of a collection in the Department of Anatomy of Akita University, Akita, Japan. The foetuses had been donated by their families to the Department from 1975 to 1985 and had been preserved in $10 \%$ $\mathrm{w} / \mathrm{w}$ neutral formalin solution for more than 30 years. The available data were limited to the date of donation and the gestational age; family name, the names of the obstetrician and hospital and the reason for abortion were not available. The use of these samples for research was approved by the Akita University Ethics Committee (No. 1428).

From 200 to 300 semiserial sections, 5 microns thick at 50-100 micron intervals, covering the entire heart, were prepared from each sample. One of every 5-10 sections was stained with haematoxylin and eosin (H\&E), whereas the other sections were used for immunohistochemistry. Antibodies used were 1) mouse monoclonal anti-human CD34 (dilution, 1:100; Dako, Glostrup, Denmark; IR632); 2) mouse monoclonal anti-human alpha smooth muscle actin (SMA; 1:100; Dako M0851); and 3) rabbit polyclonal anti-human $\mathrm{S} 100$ protein (dilution, 1:100; Dako N1573). The sections were subsequently incubated for 30 min in Histofine Simple Stain Max-PO (Nichirei, Tokyo, Japan) for the diaminobenzidine (DAB) reaction with horseradish peroxidase (HRP) and counterstained with haematoxylin. Sections were monitored and photographed with a Nikon Eclipse 80, whereas photos at ultra-low magnification (objective lens less

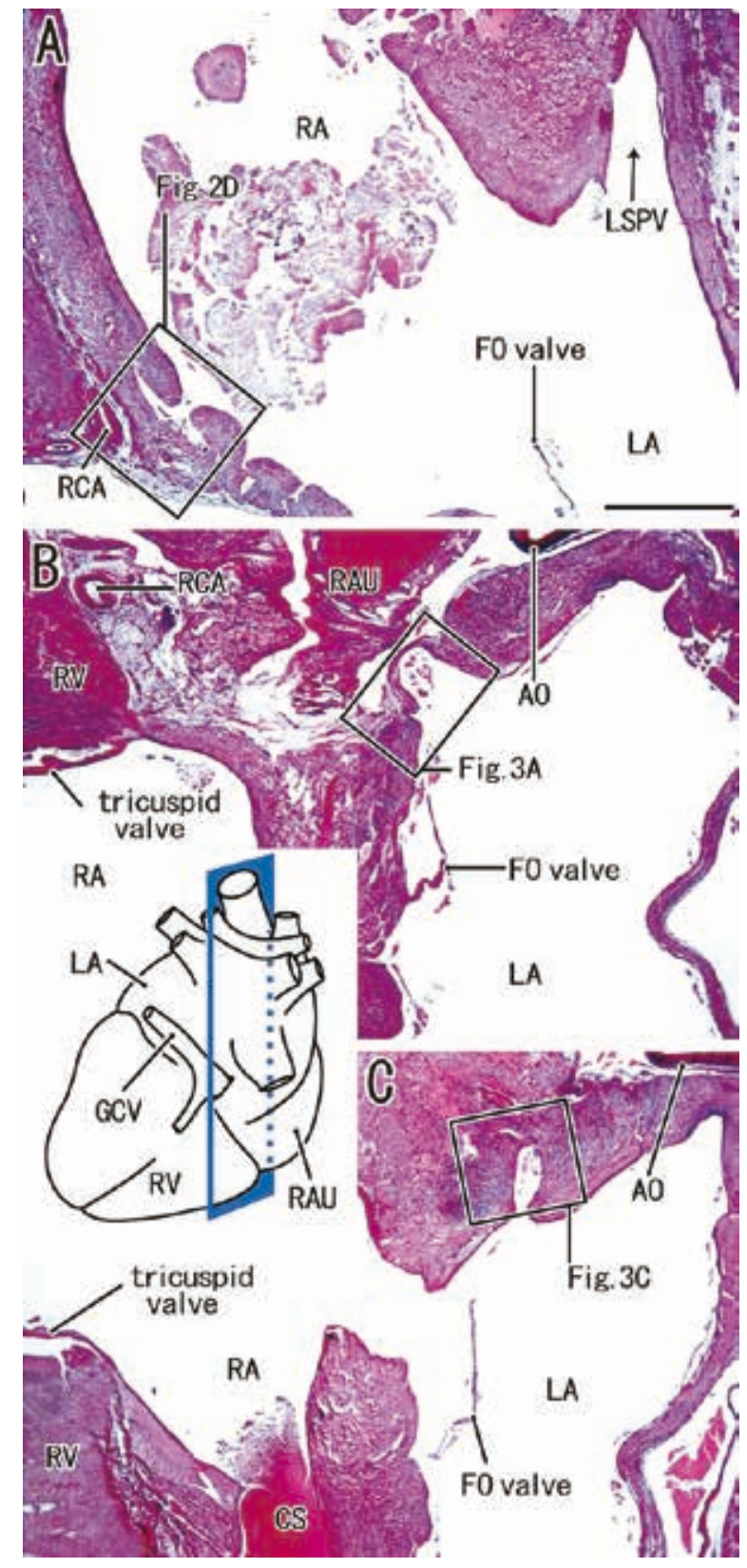

Figure 1. A-C. Topographical anatomy of veins directly draining into the atria. $A$ heart from a foetus of crown-rump length (CRL) $275 \mathrm{~mm}$. Panel A is $1.2 \mathrm{~mm}$ medial to panel B. Panel A contains a wide sinus of the right atrium (RA). Panel $B$ contains a large gulf-like sinus of the left atrium (LA), with the latter becoming narrower in panel $C$. The squares in panels $A-C$ are shown at higher magnification in Figures 2 and 3 . All panels were prepared at the same magnification (scale bar in panel A, $1 \mathrm{~mm}$ ). An insert (line-drawing) between panels $B$ and $C$ exhibits one of sagittal sectional planes used in the present study: this is similar or corresponds to sectional planes for panels A and C. However, the heart morphology in the insert (posterior view) is not foetal but adult-like for simplification; $\mathrm{AO}$ - ascending aorta; $\mathrm{CS}$ - coronary sinus; $\mathrm{FO}$ valve - valve of the foramen ovale; LA — left atrium; LSPV — left superior pulmonary vein; RA — right atrium; RAU — right auricle; RCA — right coronary artery; RV — right ventricle. 

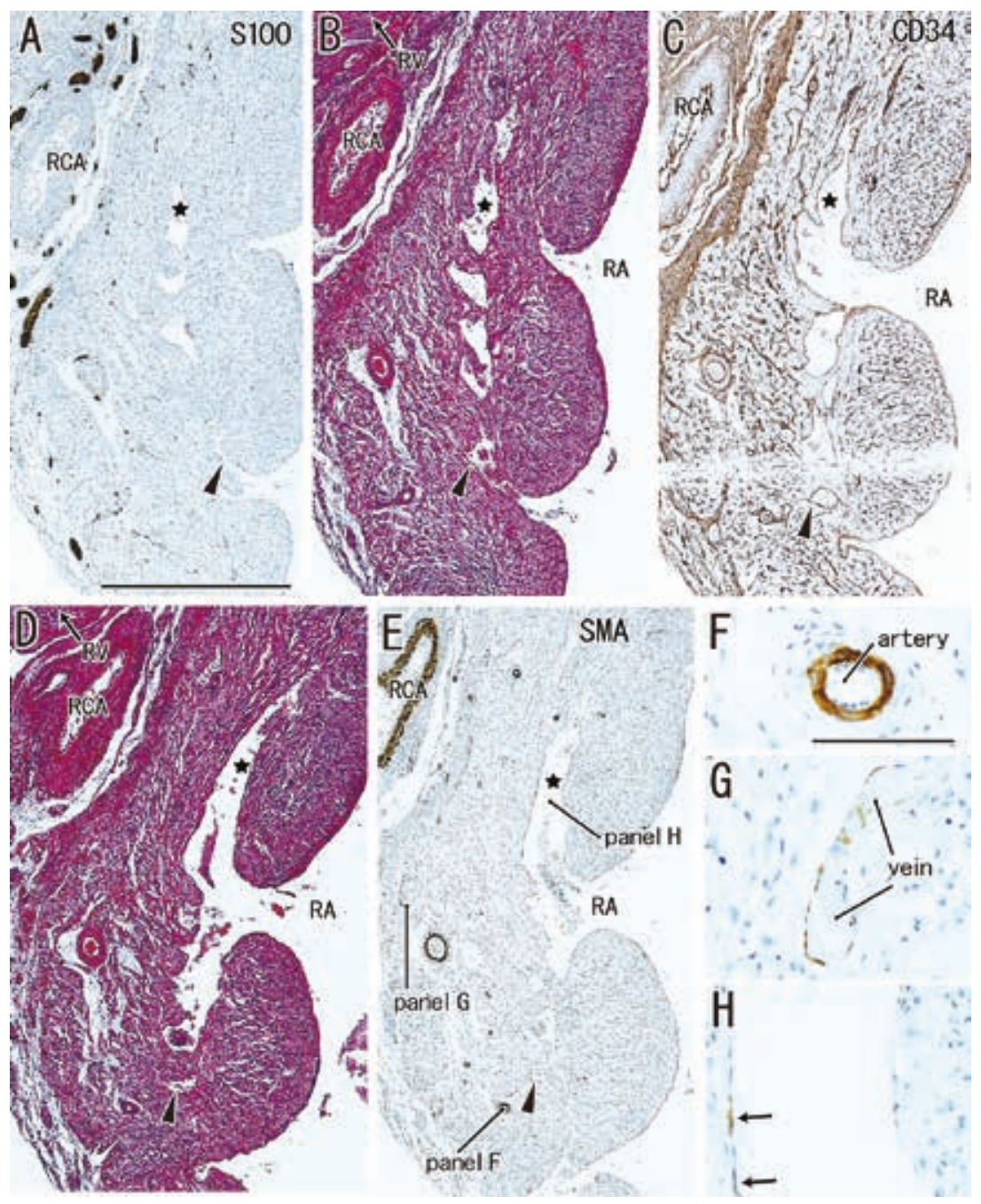

Figure 2. A-H. A vein draining directly into the right atrium. A heart from a foetus of crown-rump length (CRL) $275 \mathrm{~mm}$ (the same specimen as in Fig. 1). Sections were stained with antibodies to $S 100$ (A), CD34 (C) and smooth muscle actin (SMA) (E). Intervals between panels are $0.5 \mathrm{~mm}$. Panel $\mathrm{D}$ is a higher magnification view of a square in Figure 1A. The arrowhead and star in each panel display the same site of a vein or sinus, respectively. An opening to the right atrium (RA) is wide in panels $D$ and $E$, but the peripheral part (i.e., a directly draining vein) is narrow in the myocardium (A). Panels $\mathrm{F}-\mathrm{H}$ are higher magnification views of veins and a thin artery indicated in panel E: SMA-positive structures are limited to the endothelium $(F, G)$. A part of endothelium of the direct drainage vein also expresses reactivity of SMA (arrows in panel $H$ ). Panels A-E or $\mathrm{F}-\mathrm{H}$ were prepared at the same magnification (scale bars: $1 \mathrm{~mm}$ in panel A: $0.1 \mathrm{~mm}$ in panel $\mathrm{F}$ ); $\mathrm{RCA}$ — right coronary artery; $\mathrm{RV}$ - right ventricle.

than $\times 2$ ) were taken using a high-grade flat scanner with translucent illumination (Epson scanner GTX970).

Although CD34 is a marker of developing blood vessels (reviewed by Chang et al. [2]), this study aimed to identify fibrous structures in and around the vascular wall $[1,6]$. The antibody to SMA used in this study (Dako M0851) stained all smooth muscle cells, but in this study, it was used to detect venous endothelium $[5,10]$.

\section{RESULTS}

Semiserial sagittal sections at $50-100$ micron intervals were obtained from 14 hearts of gestational age 25-33 weeks. H\&E staining of these sections showed 75 veins draining directly into the atria. All hearts were positive for direct drainage, with a maximum of 10 veins per heart. Of these 75 veins, 55 emptied into the right atrium and 20 into the left atrium, with some hearts containing both right and left terminals (Figs. 1-3). These veins tended to be found near the auricle or the atrial septum and few were seen near orifices of the caval veins and pulmonary veins. The atrial openings of the direct draining veins were narrow; however, when traced along semiserial sections, they became wider and continuous with an intratrabecular sinus or space of the atrial wall (Figs. 1B, C; 3A, C). During a wavy 



Figure 3. A-G. A vein draining directly into the left atrium. A heart from a foetus of crown-rump length (CRL) $275 \mathrm{~mm}$ (the same specimen as in Fig. 1). Sections were stained with antibodies to $S 100(A, E), C D 34$ (F) and smooth muscle actin (SMA) (G). Intervals between panels are $0.35 \mathrm{~mm}$ $(A-B), 0.2 \mathrm{~mm}(B-C, E-F), 0.05 \mathrm{~mm}(C-D)$, and $0.1 \mathrm{~mm}(D-E, F-G)$. Panels $A$ and $C$ are higher magnification views of the squares in Figures $1 B$ and $C$, respectively. The star in each panel displays the same site of a vein or sinus. An opening of the sinus to the left atrium (LA) is wide in panel $\mathrm{A}$, but becomes narrower in panel $\mathrm{C}$ and the peripheral part (i.e., a directly draining vein) is very thin in panel $\mathrm{G}$ (arrows). The arrowheads in panels $F$ and $G$ indicate another directly draining vein. All panels were prepared at the same magnification (scale bar in panel $A, 1 \mathrm{~mm}$ )

course less than $1 \mathrm{~mm}$ peripheral to the terminal, the direct draining veins became much thinner and disappeared within the myocardium (Figs. 2A; 3G). We did not identify direct draining veins to the left or right ventricle in the present sections.

The anterior cardiac vein ended in a deep intratrabecular space or sinus (Fig. 4), while the short cardiac vein drained into the coronary sinus (Fig. 5). The anterior cardiac vein was observed near the peripheral course of the great cardiac vein. In one specimen, a branch of the great cardiac vein emptied directly into the right atrium near the terminal of the anterior cardiac vein (Fig. 4G-I). No veins were observed communicating between the anterior and great cardiac veins in the present sections although there was a limitation of observations using semiserial sections.

All of the samples were immunohistochemically positive for S100 protein. S100-positive nerves were always present along the thin branches of cardiac arteries, with a few nerves also present along the tributaries of the epicardiac and intramuscular veins. The directly draining veins were not accompanied by nerves within a distance of 50 microns from the wall. In 8 of the 14 heart specimens tributaries of the great cardiac vein were weakly and non-specifically 

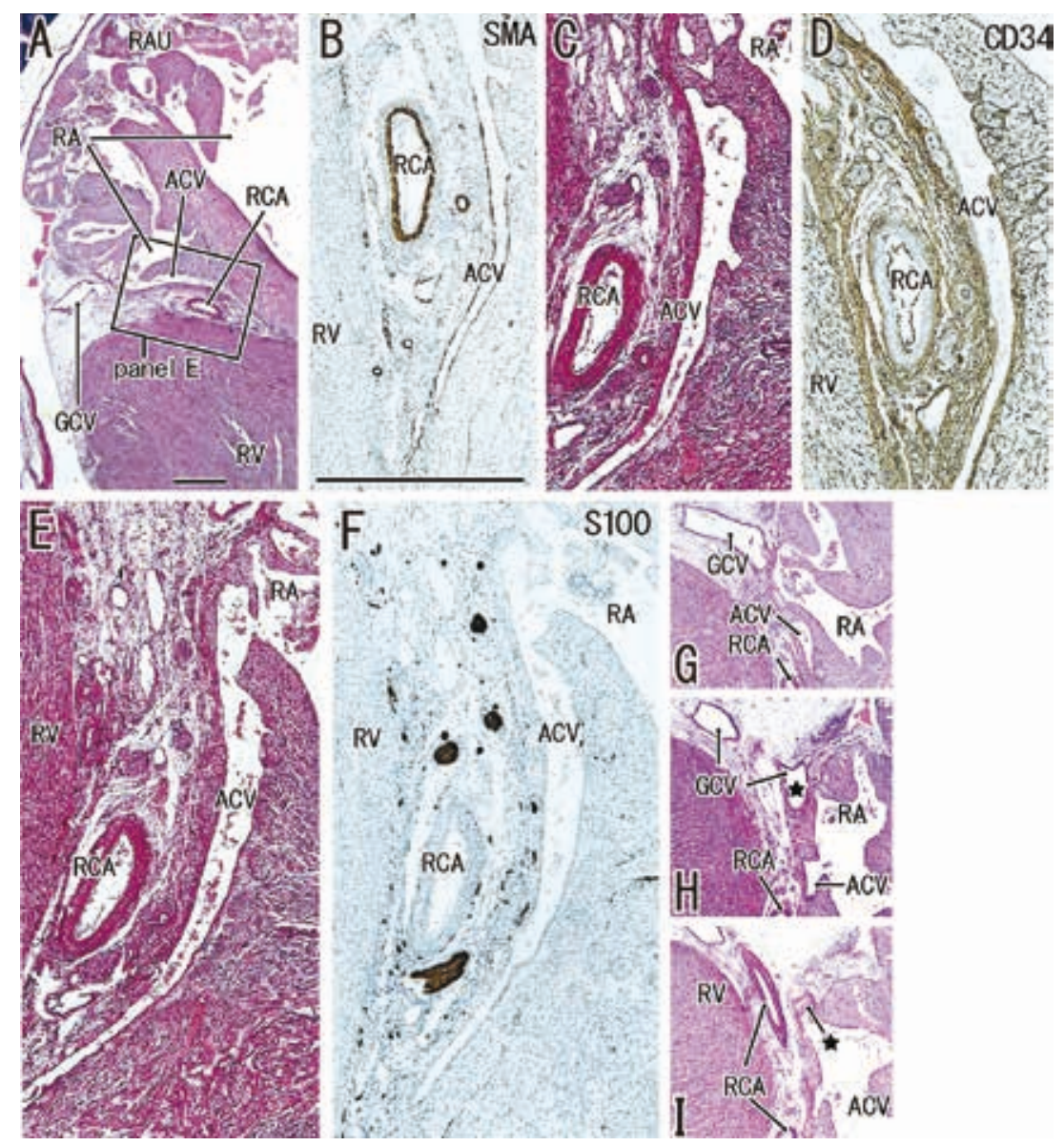

Figure 4. A-I. An anterior cardiac vein draining into the right atrium. A heart from a foetus of crown-rump length (CRL) $250 \mathrm{~mm}$. Panel A displays the topographical anatomy, including the anterior cardiac vein (ACV). Sections were stained with antibodies to S100 (F), CD34 (D) and smooth muscle actin (SMA) (B). Panel $E$ is a higher magnification view of the square in panel $A$. Intervals between panels are $0.05 \mathrm{~mm}(B-C$, $\mathrm{C}-\mathrm{D}, \mathrm{D}-\mathrm{E}, \mathrm{E}-\mathrm{F}), 0.25 \mathrm{~mm}(\mathrm{~F}-\mathrm{G}), 0.3 \mathrm{~mm}(\mathrm{G}-\mathrm{H})$ and $0.2 \mathrm{~mm}(\mathrm{H}-\mathrm{I})$, respectively. Panels $\mathrm{G}-\mathrm{I}$ show a branch (star) of the great cardiac vein (GCV) draining directly into the right atrium (RA) near the opening of the anterior cardiac vein. Panels B-I were prepared at the same magnification (scale bars in panels A and B: $1 \mathrm{~mm}$ ); RAU — right auricle; RCA — right coronary artery; RV — right ventricle.

positive for CD34 and SMA, possibly due to long preservation of these specimens in formalin solution.

Figures $2-5$ show some of our observations in 6 of these hearts. Direct draining veins were negative for SMA (Figs. 2E; 3G) except for a limited short part expressing reactivity of SMA (Fig. $2 \mathrm{H}$ ). In contrast, the positive reaction of the endothelia was seen in the other veins in the myocardium (Fig. 2G) as well as tributaries of the great cardiac vein (e.g., the middle cardiac vein; Fig. 5B). No or few CD34-positive fibres were seen in, along and around the walls of the directly draining veins (Figs. 2C; 3F), in contrast to tributaries of the great cardiac vein (e.g., the middle cardiac vein; Fig. 5E). The endothelia of the small (Fig. 5B) and anterior (Fig. 4B) cardiac veins were immunohistochemically positive for SMA expression, except at the terminal portion near the coronary sinus or right atrium. Moreover, abundant numbers of CD34-positive fibres were observed in the venous walls of these two veins (Figs. 4D; 5F).

In spite of the present long preservation, the positive reaction of SMA in the venous endothelia was just same as in our previous studies using short-preserved foetuses [10]. In addition, SMA expression was seen also in media of thick arteries (e.g., the right coronary artery; Figs. 2E; 4B). The endocardium and $a$ thin tissue layer beneath the endocardium were negative or weakly positive for both SMA and CD34. The myocardium contained a meshwork of abundant CD34-positive fibres. Beneath the endocardium, a thick CD34-positive plate was seen, possibly corresponding to a border between the epicardium and myocardium (Fig. $2 \mathrm{C} ; 3 \mathrm{3F}$ ). The smooth muscle layer of the coronary artery had not yet been established (e.g., the right coronary artery; Figs. 2E; 4B). 


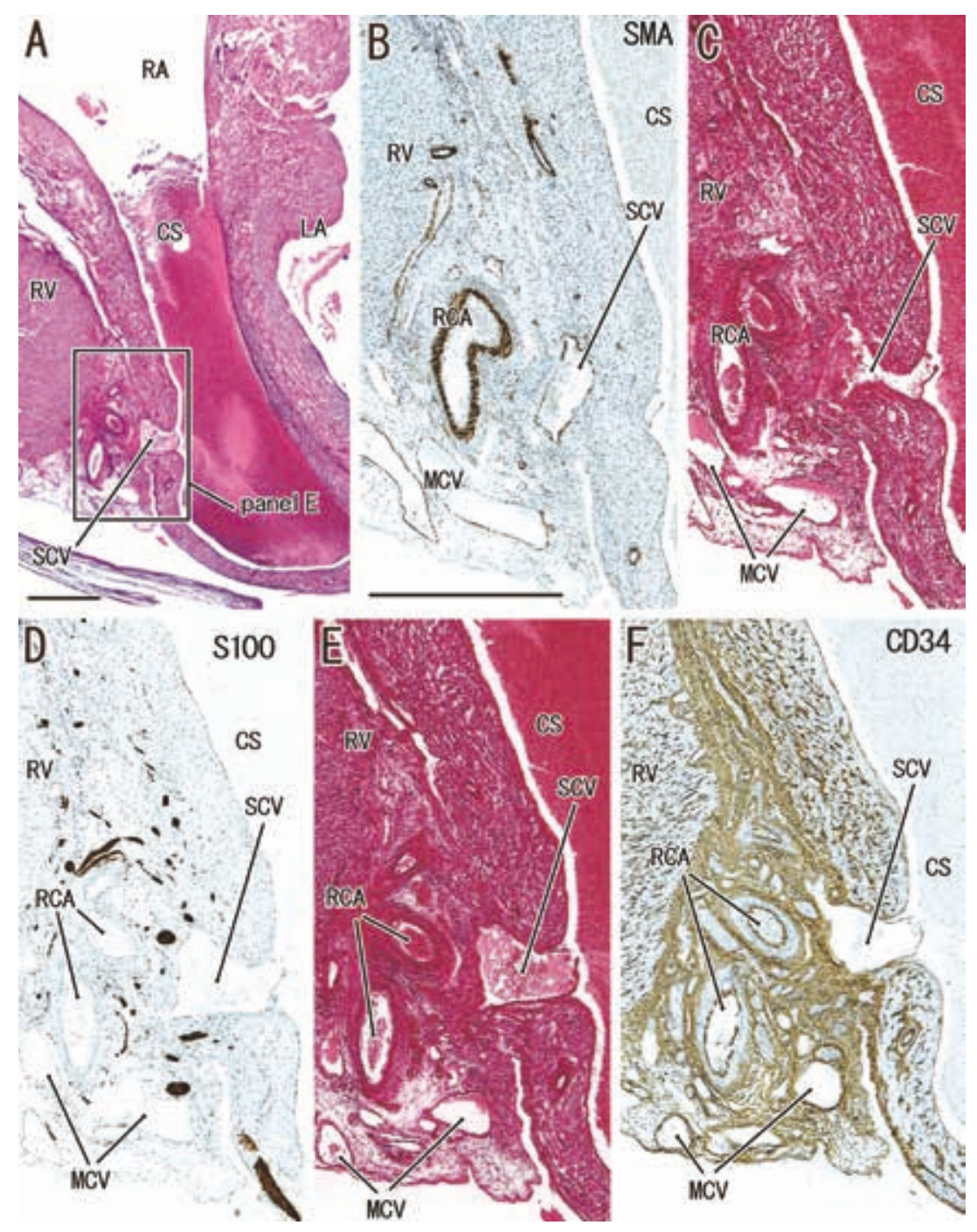

Figure 5. A small cardiac vein draining into the coronary sinus. A heart from a foetus of crown-rump length (CRL) $265 \mathrm{~mm}$. Panel A displays the topographical anatomy, including the short cardiac veins (SCV). Sections were stained with antibodies to S100 (D), CD34 (F) and smooth muscle actin (SMA) (B). Panel $E$ is a higher magnification view of the square in panel $A$. Intervals between panels are $0.05 \mathrm{~mm}$ (B-C, C-D, $\mathrm{D}-\mathrm{E}, \mathrm{E}-\mathrm{F})$. The immunoreactivity of the middle cardiac vein (MCV) to anti-SMA and anti-CD34 was typical of veins. Panels B-F were prepared at the same magnification (scale bars in panels A and B, $1 \mathrm{~mm}$ ); CS — coronary sinus; RA — right atrium; RCA — right coronary artery; RV — right ventricle.

\section{DISCUSSION}

Veins originating from the left ventricular wall "cross" the great cardiac vein superficially without any communication and empty directly into the adjacent left atrium [18]. These morphologic findings strongly suggest that the origin of these direct drainage veins differ from the origin of the great cardiac vein and its tributaries. The present study demonstrated that, in late-stage human foetuses, a gulf-like intertrabecular sinus or space in the atrium narrowed at the margins and continued to a thin vein originating from the myocardium. The developing myocardium and possibly its contrac- tion may make the gulf even narrower. Notably, the thin vein (i.e., a developing direct drainage vein) was negative for both CD34 and SMA, in contrast to tributaries of the great cardiac vein. Because of the thinness and short course of the direct veins, they might not connect with the doubly negative veins at least in the late stage foetuses. This negativity suggests that the vein was immature and developed much later than the tributaries of the great cardiac vein. It seemed unlikely that these directly draining veins had developed from the early epicardiac vascular plexus connecting to the coronary sinus. In addition, although the cardiac vein was not always 
accompanied by nerves, the directly draining veins were distant from nerve twigs in the myocardium.

People may suspect that the present direct drainage veins were likely to be peripheral parts of the intertrabecular sinus or spaces. However, the direct veins reached the myocardium according to a mesh-like configuration of CD34-positive fibres. According to interpretations by von Lüdinghausen [18], the direct veins (his smallest cardiac vessels) represent the primitive, embryonic sinusoidal circulation. However, we did not found such a morphology in previous studies of the right and left atria using many midterm foetuses [7, 11]. Other than primitive and embryonic, the intertrabecular sinus might be involved into the myocardium after midterm. We did not find SMA-positive endothelium in most parts of the direct vein as well as the atrial endocardium. As Robb [14] concluded, the endothelia of the direct vein seemed to be originated and extended from the atrial endocardium.

The anterior cardiac veins, usually 2 or 3 but sometimes as many as 5 in number, drain directly into the (right) atrium. The epicardial course of the anterior vein is long, crossing the right coronary artery in the atrioventricular sulcus. In contrast, the course of the direct veins from the myocardium to the atria was short. Moreover, the anterior veins were positive for both CD34 and SMA. Therefore, like tributaries of the great cardiac vein, the anterior cardiac veins may develop from the epicardiac vascular plexus. One of the anterior cardiac veins often joins the great cardiac vein in variable combinations [9]. We found that a branch of the great cardiac vein emptied into a deep intertrabecular sinus of the right atrium near the terminal of the anterior cardiac vein (Fig. 4G-I). An anastomosis between the anterior and great cardiac veins may develop secondarily through the involvement of the sinus with the vein.

\section{Acknowledgements}

This study was supported in part by a Grant-in-Aid for Scientific Research (JSPS KAKENHI No. 16K08435) from the Ministry of Education, Culture, Sports, Science and Technology in Japan.

\section{REFERENCES}

1. Abe Si, Suzuki M, Cho KHo, et al. CD34-positive developing vessels and other structures in human fetuses: an immunohistochemical study. Surg Radiol Anat. 2011; 33(10): 919-927, doi: 10.1007/s00276-011-0854-2, indexed in Pubmed: 21789504.
2. Chang $\mathrm{H}$, Cho KHo, Hayashi $\mathrm{S}$, et al. Site- and stagedependent differences in vascular density of the human fetal brain. Childs Nerv Syst. 2014; 30(3): 399-409, doi: 10.1007/s00381-013-2272-8, indexed in Pubmed: 24005801.

3. Grant RT. Development of the cardiac coronary vessels in the rabbit. Heart. 1926; 13: 261-271.

4. Grant RT, Regnier M. The comparative anatomy of the cardiac coronary vessels. Heart. 1926; 13: 285-317.

5. Hayashi S, Murakami G, Ohtsuka A, et al. Connective tissue configuration in the human liver hilar region with special reference to the liver capsule and vascular sheath. J Hep Bil Pancr Surg. 2008; 15(6): 640-647, doi: 10.1007/ s00534-008-1336-8, indexed in Pubmed: 18987936.

6. Katori $\mathrm{Y}$, Kiyokawa H, Kawase $\mathrm{T}$, et al. CD34-positive primitive vessels and other structures in human fetuses: an immunohistochemical study. Acta Otolaryngol. 2011; 131(10): 1086-1090, doi: 10.3109/00016489.2011.590 152, indexed in Pubmed: 21651317.

7. Kim JiH, Hwang SiE, Rodríguez-Vázquez JF, et al. Upper terminal of the inferior vena cava and development of the heart atriums: a study using human embryos. Anat Cell Biol. 2014; 47(4): 236-243, doi: 10.5115/ acb.2014.47.4.236, indexed in Pubmed: 25548721.

8. Mechanik N. Das Venensystem der Herzwände. Zeitschrift für Anatomie und Entwicklungsgeschichte. 1934; 103(6): 813-843, doi: 10.1007/bf02118755.

9. Mierzwa J, Kozielec T. Variation of the anterior cardiac veins. Folia Morphol. 1975; 34(2): 125-133, indexed in Pubmed: 1080721.

10. Miyake N, Hayashi S, Kawase T, et al. Fetal anatomy of the human carotid sheath and structures in and around it. Anat Rec (Hoboken). 2010; 293(3): 438-445, doi: 10.1002/ar.21089, indexed in Pubmed: 20169562.

11. Naito M, Yu HC, Kim JiH, et al. Topographic anatomy of the fetal inferior vena cava, coronary sinus, and pulmonary veins: Variations in Chiari's network. Clin Anat. 2015; 28(5): 627-637, doi: 10.1002/ca.22489, indexed in Pubmed: 25425533.

12. Ortale JR, Marquez CQ. Anatomy of the intramural venous sinuses of the right atrium and their tributaries. Surg Radiol Anat. 1998; 20(1): 23-29, indexed in Pubmed: 9574485.

13. Ortale JR, Gabriel EA, lost C, et al. The anatomy of the coronary sinus and its tributaries. Surg Radiol Anat. 2001; 23(1): 15-21, indexed in Pubmed: 11370136.

14. Robb JS. Comparative basic cardiology. Grune \& Stratton, New York. 1965: 123-140.

15. Steding G, Seidl W. Cardiovaskuläres System. In Human Embryologie. Hinrichsen KV (ed). Springer, Berlin. 1990: 205-294.

16. Vobořil Z, Schiebler TH. Über die Entwicklung der Gefäßversorgung des Rattenherzens. Z Anat EntwGesch. 1969; 129(1): 24-40, doi: 10.1007/bf00521953.

17. von Lüdinghausen $M$, Ohmachi $N$, Besch $S$, et al. Atrial veins of the human heart. Clin Anat. 1995; 8(3): 169-189, doi: 10.1002/ca.980080302, indexed in Pubmed: 7606591.

18. von Lüdinghausen $M$. The venous drainage of the human myocardium. Adv Anat Embryol Cell Biol. 2003; 168(I-VIII): 1-104, indexed in Pubmed: 12645157. 